\title{
AREA OF THE COMPLEMENT OF THE FAST ESCAPING SETS OF A FAMILY OF ENTIRE FUNCTIONS
}

\author{
SONG ZHANG AND FEI YANG
}

\begin{abstract}
Let $f$ be an entire function with the form $f(z)=P\left(e^{z}\right) / e^{z}$, where $P$ is a polynomial with $\operatorname{deg}(P) \geq 2$ and $P(0) \neq 0$. We prove that the area of the complement of the fast escaping set (hence the Fatou set) of $f$ in a horizontal strip of width $2 \pi$ is finite. In particular, the corresponding result can be applied to the sine family $\alpha \sin (z+\beta)$, where $\alpha \neq 0$ and $\beta \in \mathbb{C}$.
\end{abstract}

\section{INTRODUCTION}

Let $f: \mathbb{C} \rightarrow \mathbb{C}$ be a transcendental entire function. Denote by $f^{\circ n}$ the $n$ th iterate of $f$. The Fatou set $F(f)$ of $f$ is defined as the maximal open set in which the family of iterates $\left\{f^{\circ n}: n \in \mathbb{N}\right\}$ is normal in the sense of Montel. The complement of $F(f)$ is called the Julia set of $f$, which is denoted by $J(f)$. It is well known that $J(f)$ is a perfect completely invariant set which is either nowhere dense or coincides with $\mathbb{C}$. For more details about these sets, one can refer Bea91, CG93 and Mil06 for rational maps, and Ber93 and EL92 for meromorphic functions.

Already in 1920s, Fatou considered the iteration of transcendental entire functions [Fat26] and one of his study object was $f(z)=\alpha \sin (z)+\beta$, where $0<\alpha<1$ and $\beta \in \mathbb{R}$. After Misiurewicz showed that the Fatou set of $f(z)=e^{z}$ is empty in 1981 Mis81, the dynamics of exponential maps and trigonometric functions attracted many interests from then on. See [DK84, DT86 and DG87 for example. In particular, in $1987 \mathrm{McMullen}$ McM87 proved a remarkable result which states that the Julia set of $\sin (\alpha z+\beta), \alpha \neq 0$ always has positive Lebesgue area and the Hausdorff dimension of the Julia set of $\lambda e^{z}, \lambda \neq 0$ is always 2. From then on a series of papers considered the area and the Hausdorff dimension of the dynamical objects of the transcendental entire functions, not only for the Julia sets in dynamical planes (see [Sta91, Kar99a, Kar99b, Tan03, Sch07, Bar08, RS10, AB12, Rem14, Six15a and the references therein for example), but also the bifurcation loci in the parameter spaces (see Qiu94 and [ZL12]).

Unlike the polynomials, the Julia set of a transcendental entire function $f$ is always unbounded. Since the Fatou set of $f$ is dense in the complex plane (if $F(f) \neq \emptyset)$, it is interesting to ask when the Fatou set of $f$ has finite area. For the sine function $f(z)=\sin z$, Milnor conjectured that the area of the Fatou set of $f$ is finite in a vertical strip of width $2 \pi$. By applying the tools in [McM87, Schubert proved this conjecture in 2008 Sch08.

For a transcendental entire function $f$, the escaping set $I(f)$ was studied firstly by Eremenko in [Ere89. A subset of the escaping set, called the fast escaping set $A(f)$, was introduced by Bergweiler and Hinkkanen in BH99. These sets have received quite a lot of attention recently. Especially for the fast escaping set, see Six11, RS12, Six13, Six15b, Evd16] and the references therein. In this paper,

Date: May 11, 2022.

2010 Mathematics Subject Classification. Primary: 37F45; Secondary: 37F10, 37F25.

Key words and phrases. Fatou set; Julia set; Lebesgue area. 
we consider the area of the complement of the fast escaping sets of a family of entire functions and try to extend the result of Schubert to this class. Our main result is the following.

Theorem 1.1. Let $P$ be a polynomial with $\operatorname{deg}(P) \geq 2$ and $P(0) \neq 0$. Then the area of the complement of the fast escaping set of any function with the form $f(z)=P\left(e^{z}\right) / e^{z}$ is finite in any horizontal strip of width $2 \pi$.

The method in this paper is strongly inspired by the work of McMullen and Schubert (McM87] and [Sch08]). It is worth to mention that we give also a specific formula of the upper bound of $\operatorname{Area}\left(S \cap A(f)^{c}\right)$ in terms of the coefficients of the polynomial $P$ (see Theorem 3.1), where $S$ is any horizontal strip of width $2 \pi$ and $A(f)^{c}$ is the complement of the fast escaping set of $f$. In fact, we believe that our method can be adopted also to the type of entire functions with the form

$$
f(z)=\frac{P(w)}{w^{m}} \circ \exp (z)
$$

completely similarly, where $m \geq 1$ is a positive integer, $P$ is a polynomial with degree $\operatorname{deg}(P) \geq m+1$ and $P(0) \neq 0$.

As a consequence of Theorem 1.1 and Theorem 3.1, we have the following result on the area of the complement of the fast escaping set of the sine family.

Theorem 1.2. Let $S$ be any vertical strip of width $2 \pi$. Then the area of the complement of the fast escaping set of $f(z)=\alpha \sin (z+\beta)$ with $\alpha \neq 0$ satisfies

$$
\operatorname{Area}\left(S \cap A(f)^{c}\right) \leq(4 \pi+4 r)\left(x^{*}+r+8 c e^{4-x^{*} / 2} \frac{r}{1-e^{-r / 2}}\right),
$$

where

$$
r=\frac{1}{8}, \quad c=\frac{536 \sqrt{2}}{|\alpha|}+\frac{1}{|\alpha|^{2}}
$$

and

$$
x^{*}=\max \left\{\log \left(1+\frac{18 K}{|\alpha|}\right), \log \left(\frac{8(K+1)}{|\alpha|}\right), 6 \log 2,12+2 \log c\right\}
$$

with $K=\max \{|\alpha| / 2,|\beta|\}$. In particular, if $f(z)=\sin z$ or $\cos z$, then

$$
\text { Area }\left(S \cap A(f)^{c}\right)<361 \text {. }
$$

Since the fast escaping set of $f(z)=P\left(e^{z}\right) / e^{z}$ is contained in the Julia set (see Corollary 2.11, it means that the complement of the fast escaping set contains the Fatou set and hence Theorem 1.2 is a generalization of Schubert's result. In [Sch08] Schubert proved that $\operatorname{Area}(S \cap F(f))<574$ for $f(z)=\sin z$, where $S$ is a vertical strip with width $2 \pi$. See Figure 1 .

We collect some notations which will be used throughout of this paper. Let $\mathbb{N}$, $\mathbb{Z}, \mathbb{R}$ and $\mathbb{C}$, respectively, be the set of natural numbers, integers, real numbers and complex numbers. For any $x \geq 0$, we use $[x]$ to denote the integer part of $x$. Hence $x-1<[x] \leq x$. For a subset $X$ of $\mathbb{C}$, we use $X^{c}$ to denote the complement of $X$ in $\mathbb{C}$. All the distance and diameter in this paper are measured in the Euclidean metric and the area is regarded as the two-dimensional planar Lebesgue area. We use $\mathbb{D}(a, r):=\{z \in \mathbb{C}:|z-a|<r\}$ to denote the round disk with center $a \in \mathbb{C}$ and radius $r>0$.

Acknowledgements. This work is supported by the National Natural Science Foundation of China (grant Nos. 11671092, 11671191) and the Fundamental Research Funds for the Central Universities (grant No.0203-14380013). We would like to thank Lasse Rempe-Gillen for valuable comments which improved the statements of the main results in this paper and Liangwen Liao for helpful conversations. 

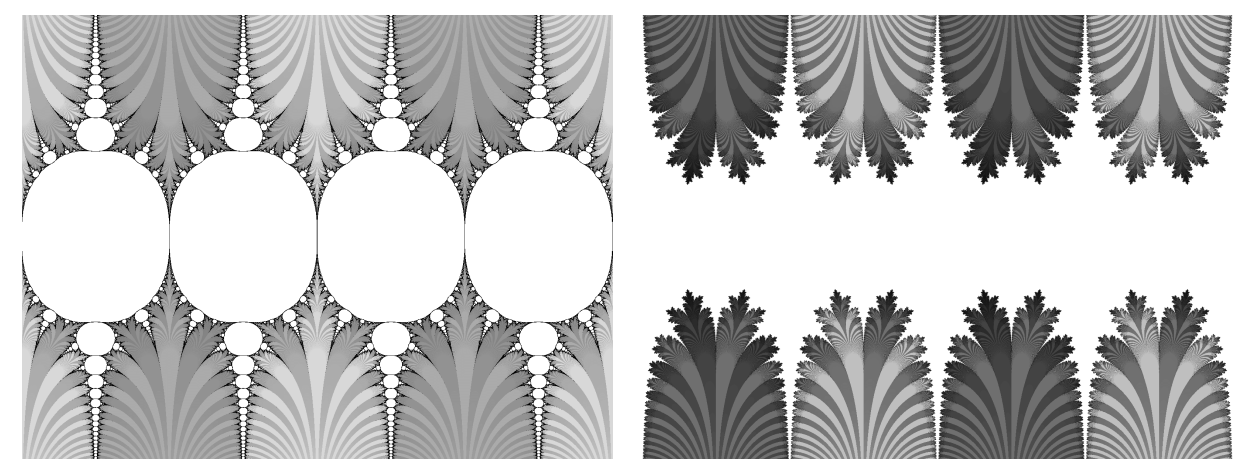

Figure 1: The Fatou sets (white regions) of $f(z)=\sin z$ and $f(z)=\cos z$. Both of these functions have period $2 \pi$. It is shown in Theorem 1.2 that the area of the complement of the fast escaping set (hence the Fatou set) of $f$ in a vertical strip with width of $2 \pi$ is bounded above by 361 .

\section{Distortion Lemmas And SOME BASIC SETtings}

2.1. Distortion quantities. As in McM87 and Sch08, we introduce some quantities of distortion in this subsection. Let $D$ be a bounded set in the complex plane $\mathbb{C}$ and let $f$ be a holomorphic function defined in a neighbourhood of $D$. We say that $f$ has bounded distortion on $D$ if there are positive constants $c$ and $C$, such that for all distinct $x$ and $y$ in $D$, one has

$$
c<\frac{|f(x)-f(y)|}{|x-y|}<C .
$$

The quantity

$$
L\left(\left.f\right|_{D}\right):=\inf \{C / c: c \text { and } C \text { satisfy (1) }\}
$$

is the distortion of $f$ on $D$. By (1) we have

$$
\sup _{z \in D}\left|f^{\prime}(z)\right| \leq C \quad \text { and } \quad \inf _{z \in D}\left|f^{\prime}(z)\right| \geq c .
$$

Therefore, $L\left(\left.f\right|_{D}\right)$ has a lower bound satisfying

$$
L\left(\left.f\right|_{D}\right) \geq \frac{\sup _{z \in D}\left|f^{\prime}(z)\right|}{\inf _{z \in D}\left|f^{\prime}(z)\right|} .
$$

The equality holds in this inequality if $D$ is a convex domain.

Let $\operatorname{Area}(E)$ be the Lebesgue area of the measurable set $E \subset \mathbb{C}$. If $X$ and $D$ are two measurable subsets of the complex plane with $\operatorname{Area}(D)>0$, we use

$$
\operatorname{density}(X, D):=\frac{\operatorname{Area}(X \cap D)}{\operatorname{Area}(D)}
$$

to denote the density of $X$ in $D$. If $c$ and $C$ satisfy (1), then $c^{2} \operatorname{Area}(X) \leq$ Area $(f(X)) \leq C^{2} \operatorname{Area}(X)$. This means that

$$
\operatorname{density}(f(X), f(D)) \leq L\left(\left.f\right|_{D}\right)^{2} \operatorname{density}(X, D) .
$$

The nonlinearity of $f$ on $D$ is defined as

$$
N\left(\left.f\right|_{D}\right):=\sup \left\{\frac{\left|f^{\prime \prime}(z)\right|}{\left|f^{\prime}(z)\right|}: z \in D\right\} \cdot \operatorname{diam}(D),
$$

provided the right-hand side is finite. In the following by square we mean a closed square whose sides are parallel to the coordinate axes. We will use the following relation between the distortion and nonlinearity on squares. 
Lemma 2.1. Let $Q$ be a compact and convex domain in $\mathbb{C}$ (in particular if $Q$ is a square) and let $f$ be a conformal map defined in a neighbourhood of $Q$ with $N\left(\left.f\right|_{Q}\right)<1$. Then

$$
L\left(\left.f\right|_{Q}\right) \leq 1+2 N\left(\left.f\right|_{Q}\right)
$$

Proof. Since $f$ is conformal, let $z_{0}$ be a point in $Q$ such that

$$
\left|f^{\prime}\left(z_{0}\right)\right|=\sup _{z \in Q}\left|f^{\prime}(z)\right|>0 .
$$

Since $Q$ is convex, for any $z \in Q$ we have

$$
\begin{aligned}
\frac{\left|f^{\prime}(z)-f^{\prime}\left(z_{0}\right)\right|}{\left|f^{\prime}\left(z_{0}\right)\right|} & =\frac{\left|\int_{z_{0}}^{z} f^{\prime \prime}(\zeta) d \zeta\right|}{\left|f^{\prime}\left(z_{0}\right)\right|} \leq \frac{\sup _{z \in Q}\left|f^{\prime \prime}(z)\right|}{\left|f^{\prime}\left(z_{0}\right)\right|} \cdot\left|z-z_{0}\right| \\
& \leq \sup _{z \in Q}\left\{\frac{\left|f^{\prime \prime}(z)\right|}{\left|f^{\prime}(z)\right|}\right\} \cdot \operatorname{diam}(Q)=N\left(\left.f\right|_{Q}\right)<1 .
\end{aligned}
$$

Therefore, the image of $Q$ under $f^{\prime}(z)$ is contained in the disk $\mathbb{D}\left(f^{\prime}\left(z_{0}\right),\left|f^{\prime}\left(z_{0}\right)\right|\right)$ and hence $\log f^{\prime}(z)$ is well-defined on $Q$.

Since $Q$ is compact, let $z_{1} \in Q$ such that

$$
\left|f^{\prime}\left(z_{1}\right)\right|=\inf _{z \in Q}\left|f^{\prime}(z)\right|>0 \text {. }
$$

Since $Q$ is convex and $\log f^{\prime}(z)$ is well-defined, we have

$$
\begin{aligned}
\log L\left(\left.f\right|_{Q}\right) & =\log \frac{\left|f^{\prime}\left(z_{0}\right)\right|}{\left|f^{\prime}\left(z_{1}\right)\right|} \leq\left|\log f^{\prime}\left(z_{1}\right)-\log f^{\prime}\left(z_{0}\right)\right| \\
& =\left|\int_{z_{0}}^{z_{1}}\left(\log f^{\prime}(z)\right)^{\prime} d z\right|=\left|\int_{z_{0}}^{z_{1}} \frac{f^{\prime \prime}(z)}{f^{\prime}(z)} d z\right| \\
& \leq \sup _{z \in Q}\left\{\frac{\left|f^{\prime \prime}(z)\right|}{\left|f^{\prime}(z)\right|}\right\} \cdot \operatorname{diam}(Q)=N\left(\left.f\right|_{Q}\right) .
\end{aligned}
$$

Since $e^{x} \leq 1+2 x$ for $x \in[0,1)$, we have

$$
L\left(\left.f\right|_{Q}\right) \leq \exp \left(N\left(\left.f\right|_{Q}\right)\right) \leq 1+2 N\left(\left.f\right|_{Q}\right) .
$$

Remark. McMullen notes in McM87] that $L\left(\left.f\right|_{Q}\right)$ is bounded above by $1+$ $O\left(N\left(\left.f\right|_{Q}\right)\right)$ if $N\left(\left.f\right|_{Q}\right)$ is small. After that Schubert states in Sch08] that $L\left(\left.f\right|_{Q}\right) \leq$ $1+8 N\left(\left.f\right|_{Q}\right)$ if $N\left(\left.f\right|_{Q}\right)<1 / 4$ but without a proof.

Let $n$ be a positive integer. For each $1 \leq i \leq n$, let $D_{i} \subset \mathbb{C}$ be an open set and $f_{i}: D_{i} \rightarrow \mathbb{C}$ a conformal map. Let $\sigma$ and $M$ be two positive constants satisfying

$$
\left|f_{i}^{\prime}(z)\right|>\sigma>1 \quad \text { and } \quad \frac{\left|f_{i}^{\prime \prime}(z)\right|}{\left|f_{i}^{\prime}(z)\right|}<M, \quad \text { where } z \in D_{i} \text { and } 1 \leq i \leq n \text {. }
$$

Furthermore, let $Q_{i} \subset D_{i}, 1 \leq i \leq n$ be squares with sides of length $r>0$ satisfying $Q_{i+1} \subset f_{i}\left(Q_{i}\right)$ for all $1 \leq i \leq n-1$. Define $V:=f_{n}\left(Q_{n}\right)$ and

$$
F:=\left(f_{n} \circ \cdots \circ f_{1}\right)^{-1}: V \rightarrow Q_{1} .
$$

Then $F$ is a conformal map. McMullen proved that the distortion of $F$ on $V$ is bounded above by a constant depending only on $\sigma, M$ and $r$, but not on $f_{i}$ and $n$ ([McM87]). Actually, this upper bound can be formulated in the following lemma.

Lemma 2.2. If the sides of length $r$ of $Q_{i}$ is chosen such that $r \leq 1 /(4 M)$ for all $1 \leq i \leq n$, then the distortion of $F$ on $V$ satisfies

$$
L\left(\left.F\right|_{V}\right) \leq \exp \left(\frac{\sigma}{\sigma-1}\right)
$$


Proof. Let $g_{i}$ be the inverse of $f_{i}$ which maps $f_{i}\left(Q_{i}\right)$ to $Q_{i}$ for $1 \leq i \leq n$. Recall that $V=f_{n}\left(Q_{n}\right)$. Define $V_{i}:=g_{i} \circ \cdots \circ g_{n}(V)$, where $1 \leq i \leq n$. In particular, $V_{n}=g_{n}(V)=Q_{n}$. Since $\left|f_{i}^{\prime}(z)\right|>\sigma>1$ for all $1 \leq i \leq n$, we have

$$
\operatorname{diam}\left(V_{i}\right) \leq \frac{\sqrt{2} r}{\sigma^{n-i}}, \quad \text { for all } 1 \leq i \leq n .
$$

Note that $V_{i} \subset Q_{i} \subset D_{i}$ for $1 \leq i \leq n$ since $Q_{i+1} \subset f_{i}\left(Q_{i}\right)$ for all $1 \leq i \leq n-1$. This means that there exists a square $Q_{i}^{\prime} \subset Q_{i}$ such that $V_{i} \subset Q_{i}^{\prime}$ and the length of the sides of $Q_{i}^{\prime}$ is at most $\sqrt{2} r / \sigma^{n-i}$. Hence by (4), the nonlinearity of $f_{i}$ on $Q_{i}^{\prime}$ satisfies

$$
N\left(\left.f_{i}\right|_{Q_{i}^{\prime}}\right)=\left(\sup _{z \in Q_{i}^{\prime}} \frac{\left|f_{i}^{\prime \prime}(z)\right|}{\left|f_{i}^{\prime}(z)\right|}\right) \cdot \operatorname{diam}\left(Q_{i}^{\prime}\right) \leq \frac{2 M r}{\sigma^{n-i}} \leq \frac{1}{2} .
$$

By Lemma 2.1, we have

$$
L\left(\left.f_{i}\right|_{Q_{i}^{\prime}}\right) \leq 1+\frac{4 M r}{\sigma^{n-i}}, \quad \text { for all } 1 \leq i \leq n .
$$

For any holomorphic functions $f$ and $g$, it is straightforward to verify that the distortion of $f$ and $g$ satisfies 1

$$
L\left(\left.f\right|_{V}\right)=L\left(\left.f^{-1}\right|_{f(V)}\right) \quad \text { and } \quad L\left(\left.(g \circ f)\right|_{V}\right) \leq L\left(\left.f\right|_{V}\right) L\left(\left.g\right|_{f(V)}\right) .
$$

Hence, we have

$$
\begin{aligned}
& L\left(\left.F\right|_{V}\right)=L\left(\left.\left(f_{n} \circ \cdots \circ f_{1}\right)\right|_{V_{1}}\right) \\
\leq & L\left(\left.f_{1}\right|_{V_{1}}\right) L\left(\left.f_{2}\right|_{V_{2}}\right) \cdots L\left(\left.f_{n}\right|_{V_{n}}\right) \leq L\left(\left.f_{1}\right|_{Q_{1}^{\prime}}\right) L\left(\left.f_{2}\right|_{Q_{2}^{\prime}}\right) \cdots L\left(\left.f_{n}\right|_{Q_{n}^{\prime}}\right) \\
\leq & \prod_{i=0}^{n-1}\left(1+\frac{4 M r}{\sigma^{i}}\right) \leq \prod_{i=0}^{n-1}\left(1+\frac{1}{\sigma^{i}}\right) .
\end{aligned}
$$

Since $\log (1+x) \leq x$ for all $x>0$, we have

$$
L\left(\left.F\right|_{V}\right) \leq \exp \left(\sum_{i=0}^{n-1} \frac{1}{\sigma^{i}}\right)<\exp \left(\sum_{i=0}^{\infty} \frac{1}{\sigma^{i}}\right)=\exp \left(\frac{\sigma}{\sigma-1}\right) .
$$

2.2. Nesting conditions, density and area. In his proof of the existence of Julia sets of entire functions having positive area, McMullen introduced a system of compact sets which satisfies the nesting conditions [McM87]. We now recall the precise definition.

Definition (Nesting conditions). For $k \in \mathbb{N}$, let $\mathcal{E}_{k}$ be a finite collection of measurable subsets of $\mathbb{C}$, i.e. $\mathcal{E}_{k}:=\left\{E_{k, i}: 1 \leq i \leq d_{k}\right\}$, where each $E_{k, i}$ is a measurable subset of $\mathbb{C}$ and $d_{k}:=\# \mathcal{E}_{k}<+\infty$. We say that $\left\{\mathcal{E}_{k}\right\}_{k=0}^{\infty}$ satisfies the nesting conditions if $\mathcal{E}_{0}=\left\{E_{0,1}\right\}$, where $E_{0,1}$ is a compact connected measurable set and for all $k \in \mathbb{N}$,

(a) every $E_{k+1, i} \in \mathcal{E}_{k+1}$ is contained in a $E_{k, j} \in \mathcal{E}_{k}$, where $1 \leq i \leq d_{k+1}$ and $1 \leq j \leq d_{k}$

(b) every $E_{k, i} \in \mathcal{E}_{k}$ contains a $E_{k+1, j} \in \mathcal{E}_{k+1}$, where $1 \leq i \leq d_{k}$ and $1 \leq j \leq$ $d_{k+1}$

(c) $\operatorname{Area}\left(E_{k, i} \cap E_{k, j}\right)=0$ for all $1 \leq i, j \leq d_{k}$ with $i \neq j$; and

(d) there is $\rho_{k}>0$ such that for all $1 \leq i \leq d_{k}$ and $E_{k, i} \in \mathcal{E}_{k}$, we have

$$
\operatorname{density}\left(\mathcal{E}_{k+1}, E_{k, i}\right):=\operatorname{density}\left(\bigcup_{j=1}^{d_{k+1}} E_{k+1, j}, E_{k, i}\right) \geq \rho_{k} .
$$

\footnotetext{
${ }^{1}$ We suppose that the inverse of $f$ exists in the first equality.

${ }^{2}$ Note that $\mathcal{E}_{k}$ is a collection of measurable sets for $k \in \mathbb{N}$. For simplicity, sometimes we will not distinguish $\mathcal{E}_{k}$ and the union of its elements $\bigcup_{i=1}^{d_{k}} E_{k, i}$.
} 
Let $\left\{\mathcal{E}_{k}\right\}_{k=0}^{\infty}$ be a sequence satisfying the nesting conditions. Define $E:=\cap_{k=0}^{\infty} \mathcal{E}_{k}$. The following lemma was established in [McM87, Proposition 2.1].

Lemma 2.3. The density of $E$ in $E_{0,1}$ satisfies

$$
\operatorname{density}\left(E, E_{0,1}\right) \geq \prod_{k=0}^{\infty} \rho_{k}
$$

Now we give the definition of some regions which are needed in the following. For $x>0$, we define

$$
\Lambda(x):=\{z \in \mathbb{C}:|\operatorname{Re} z|>x\} .
$$

For any given $m, n \in \mathbb{Z}$ and $r>0$, we define the closed square by

$$
Q_{r}^{m, n}:=\{z \in \mathbb{C}: m r \leq \operatorname{Re} z \leq(m+1) r \text { and } n r \leq \operatorname{Im} z \leq(n+1) r\} .
$$

Let

$$
\mathcal{Q}_{r}:=\left\{Q_{r}^{m, n}: m, n \in \mathbb{Z}\right\}
$$

be a partition of $\mathbb{C}$ by the grids with sides of length $r>0$. Sometimes we write $Q_{r}^{m, n} \in \mathcal{Q}_{r}$ as $Q_{r}$ if we don't want to emphasize the superscript of $Q_{r}^{m, n}$.

Lemma 2.4. Let $Q \subset \mathbb{C}$ be a square with sides of length $r>0$ and suppose that $f$ is conformal in a neighbourhood of $Q$ with distortion $L\left(\left.f\right|_{Q}\right)<\infty$. For any $x>0$ and $z_{0} \in Q$, we have

$$
\text { Area }\left(\cup\left\{Q_{r} \in \mathcal{Q}_{r}: Q_{r} \cap(\partial f(Q) \cup(\partial \Lambda(x) \cap f(Q))) \neq \emptyset\right\}\right) \leq c r^{2},
$$

where $c=16+12 \sqrt{2} L\left(\left.f\right|_{Q}\right)\left|f^{\prime}\left(z_{0}\right)\right|$.

This lemma was established in [Sch08, Lemma 2.3] with a different coefficient $c$. For completeness we include a proof here and the argument is slightly different.

Proof. If $\gamma \subset \mathbb{C}$ is a vertical line with length $l_{1}>0$, it is clear that

$$
\#\left\{Q_{r} \in \mathcal{Q}_{r}: Q_{r} \cap \gamma \neq \emptyset\right\} \leq 4+\frac{2 l_{1}}{r} .
$$

Let $\gamma \subset \mathbb{C}$ be a continuous curve with length $l_{2}=2 \sqrt{2} k r>0$, where $k$ is a positive integer. We claim that

$$
k^{\prime}:=\#\left\{Q_{r} \in \mathcal{Q}_{r}: Q_{r} \cap \gamma \neq \emptyset\right\} \leq 4+8 k .
$$

Indeed, if $k=1$, then it is easy to see $k^{\prime} \leq 12$. Assume that $k=n$ and in this case $k^{\prime} \leq 4+8 n$. If $k=n+1$, let $\gamma(t):[0,1] \rightarrow \mathbb{C}$ be a parameterization of $\gamma$ such that the length of $\gamma\left(\left[0, t_{0}\right]\right)$ is $2 \sqrt{2} n r$ while the length of $\gamma\left(\left[t_{0}, 1\right]\right)$ is $2 \sqrt{2} r$, where $0<t_{0}<1$. Since $\gamma\left(\left[t_{0}, 1\right]\right)$ can intersect at most 8 squares while $\gamma\left(\left[0, t_{0}\right]\right)$ can intersect at most $4+8 n$ by the assumption, it follows that $k^{\prime} \leq 4+8(n+1)$ if $k=n+1$. Hence the claim (8) is proved.

For the general case, we assume that $\gamma \subset \mathbb{C}$ is a continuous curve with length $l_{3}>0$. Let $[x]$ be the integer part of $x>0$. By $[8]$, we have

$$
\#\left\{Q_{r} \in \mathcal{Q}_{r}: Q_{r} \cap \gamma \neq \emptyset\right\} \leq 4+8\left[\frac{l_{3}}{2 \sqrt{2} r}\right]+8 \leq 12+\frac{2 \sqrt{2} l_{3}}{r} .
$$

Since $f$ is a conformal map in a neighbourhood of $Q$, we conclude that $\partial f(Q)=$ $f(\partial Q)$. From $(2)$, the length of $\partial f(Q)$ satisfies

$$
\begin{aligned}
l_{4}:=\int_{\partial f(Q)}|d \xi|=\int_{\partial Q}\left|f^{\prime}(z)\right||d z| & \leq \sup _{z \in Q}\left|f^{\prime}(z)\right| \cdot 4 r \\
& \leq 4 L\left(\left.f\right|_{Q}\right)\left|f^{\prime}\left(z_{0}\right)\right| r .
\end{aligned}
$$


Similarly, the length of $\partial \Lambda(x) \cap f(Q)$ satisfies

$$
\begin{aligned}
l_{5} \leq 2 \operatorname{diam} f(Q) & \leq 2 \sup _{z \in Q}\left|f^{\prime}(z)\right| \cdot \operatorname{diam}(Q) \\
& \leq 2 \sqrt{2} L\left(\left.f\right|_{Q}\right)\left|f^{\prime}\left(z_{0}\right)\right| r .
\end{aligned}
$$

By (7), (9), 10 and (11), we have

$$
\begin{aligned}
& \#\left\{Q_{r} \in \mathcal{Q}_{r}: Q_{r} \cap(\partial f(Q) \cup(\partial \Lambda(x) \cap f(Q))) \neq \emptyset\right\} \\
\leq & \left(4+\frac{2 l_{5}}{r}\right)+\left(12+\frac{2 \sqrt{2} l_{4}}{r}\right)=16+\frac{2 l_{5}+2 \sqrt{2} l_{4}}{r} \\
\leq & 16+12 \sqrt{2} L\left(\left.f\right|_{Q}\right)\left|f^{\prime}\left(z_{0}\right)\right| .
\end{aligned}
$$

The proof is finished if we notice that the area of each $Q_{r}$ is $r^{2}$.

2.3. Basic properties of the polynomial and entire function. For $N \geq 2$, let $P$ be a polynomial with degree at least 2 which has the form

$$
P(z)=a_{0}+a_{1} z+\cdots+a_{N} z^{N},
$$

where $a_{i} \in \mathbb{C}$ for $0 \leq i \leq N$ and $a_{0} a_{N} \neq 0$. In the rest of this article, the polynomial $P$ will be fixed. We denote

$$
K:=\max \left\{\left|a_{0}\right|,\left|a_{1}\right|, \cdots,\left|a_{N}\right|\right\}>0 .
$$

Lemma 2.5. Let $\varepsilon>0$ be any given constant. The following statements hold:

(a) If $|z| \geq 1+\frac{K}{\varepsilon\left|a_{N}\right|}>1$, then

$$
\left|P(z)-a_{N} z^{N}\right| \leq \varepsilon\left|a_{N}\right||z|^{N}
$$

(b) If $|z| \leq \frac{\varepsilon\left|a_{0}\right|}{K+\varepsilon\left|a_{0}\right|}<1$, then

$$
\left|P(z)-a_{0}\right| \leq \varepsilon\left|a_{0}\right|
$$

Proof. By the definition of $K$ in $(12)$, if $|z| \geq 1+\frac{K}{\varepsilon\left|a_{N}\right|}>1$, then

$$
\left|P(z)-a_{N} z^{N}\right| \leq K\left(1+|z|+\cdots+|z|^{N-1}\right)<K \frac{|z|^{N}}{|z|-1} \leq \varepsilon\left|a_{N}\right||z|^{N} .
$$

On the other hand, if $|z| \leq \frac{\varepsilon\left|a_{0}\right|}{K+\varepsilon\left|a_{0}\right|}<1$, then

$$
\left|P(z)-a_{0}\right| \leq K\left(|z|+\cdots+|z|^{N}\right)<K \frac{|z|}{1-|z|} \leq \varepsilon\left|a_{0}\right| .
$$

Note that

$$
P(z) / z=a_{0} z^{-1}+a_{1}+\cdots+a_{N} z^{N-1}
$$

is a rational function. Let $\mathbb{D}(a, r):=\{z \in \mathbb{C}:|z-a|<r\}$ be the open disk centered at $a \in \mathbb{C}$ with radius $r>0$. For each $R>0$ and $\theta, \xi \in[0,2 \pi)$, we denote a closed domain

$$
\mathbb{U}(R, \theta, \xi):=\left\{z \in \mathbb{C}:|z| \geq R \text { and } \theta-\frac{\xi}{2} \leq \arg (z) \leq \theta+\frac{\xi}{2}\right\} .
$$

Lemma 2.6. For every $\theta \in[0,2 \pi)$, the rational function $P(z) / z$ is univalent in a neighborhood of $\mathbb{U}\left(2 R_{1}, \theta, \frac{\pi}{N-1}\right)$ and $\overline{\mathbb{D}}\left(0, R_{2} / 2\right)$, where

$$
R_{1}=1+\frac{4 K}{\left|a_{N}\right|} \quad \text { and } \quad R_{2}=\frac{\left|a_{0}\right|}{4 K+\left|a_{0}\right|} .
$$


Proof. (a) If $|z| \geq R_{1}$, by Lemma 2.5(a) we have

$$
\left|\frac{P(z)}{z}-a_{N} z^{N-1}\right| \leq \frac{1}{4}\left|a_{N}\right||z|^{N-1} .
$$

Then one can write $P(z) / z$ as

$$
P_{1}(z)=\frac{P(z)}{z}=a_{N} z^{N-1}(1+\varphi(z)),
$$

where $\varphi(z)$ is holomorphic in $\mathbb{C} \backslash\{0\}$ and $|\varphi(z)| \leq 1 / 4$ if $|z| \geq R_{1}$.

Let $w_{0} \in \mathbb{C} \backslash\{0\}$. For any $w \in \partial \mathbb{U}\left(\left|w_{0}\right| / 2, \arg \left(w_{0}\right), \pi\right)$, we have

$$
\left|w-w_{0}\right|>\frac{1}{4}\left(|w|+\left|w_{0}\right|\right)
$$

Let $g(z):=z^{N-1}$. For each $z_{0} \in \mathbb{C}$ such that $\left|z_{0}\right| \geq 2 R_{1}$, we define $w_{0}:=g\left(z_{0}\right)=$ $z_{0}^{N-1}$. Note that $g^{-1}\left(\mathbb{U}\left(\left|w_{0}\right| / 2, \arg \left(w_{0}\right), \pi\right)\right)$ consists of $N-1$ disjoint closed domains:

$$
D_{k}:=\mathbb{U}\left(2^{-1 /(N-1)}\left|z_{0}\right|, \arg \left(z_{0}\right)+\frac{2 k \pi}{N-1}, \frac{\pi}{N-1}\right),
$$

where $0 \leq k \leq N-2$. Then for $0 \leq k \leq N-2, z_{k}:=z_{0} e^{2 k \pi \mathrm{i} /(N-1)}$ is contained in the interior of $D_{k}$.

For any $z \in \partial D_{k}$ with $0 \leq k \leq N-2$, we have $z^{N-1} \in \partial \mathbb{U}\left(\left|w_{0}\right| / 2, \arg \left(w_{0}\right), \pi\right)$. Combining (13) and (14), we have

$$
\left|z^{N-1}-z_{0}^{N-1}\right|>\frac{1}{4}\left(|z|^{N-1}+\left|z_{0}\right|^{N-1}\right) \geq\left|z^{N-1} \varphi(z)-z_{0}^{N-1} \varphi\left(z_{0}\right)\right| .
$$

Define $\varphi_{1}(z):=a_{N}\left(z^{N-1}-z_{0}^{N-1}\right)$ and $\varphi_{2}(z):=P_{1}(z)-P_{1}\left(z_{0}\right)=a_{N} z^{N-1}(1+$ $\varphi(z))-a_{N} z_{0}^{N-1}\left(1+\varphi\left(z_{0}\right)\right)$. By Rouché's theorem, $\varphi_{1}(z)=0$ and $\varphi_{2}(z)=0$ have the same number of roots in each $D_{k}$, where $0 \leq k \leq N-2$. Since $\varphi_{1}(z)=0$ has exactly one root $z_{k}$ in each $D_{k}$, this means that $\varphi_{2}(z)=0$ has exactly one root in each $D_{k}$, where $0 \leq k \leq N-2$.

On the other hand, $\overline{(14}$ holds also for $w \in \partial \mathbb{U}\left(\left|w_{0}\right| / 2,-\arg \left(w_{0}\right), \pi\right)$. By Rouché's theorem again, $\varphi_{2}(z)=0$ has no root in each $-D_{k}$, where $0 \leq k \leq N-2$. By the arbitrariness of $z_{0}$, it means that $P_{1}(z)=P(z) / z$ is univalent in a neighborhood of $\mathbb{U}\left(2 R_{1}, \theta, \frac{\pi}{N-1}\right)$, where $\theta \in[0,2 \pi)$.

(b) Similarly, by Lemma 2.5(b) one can write $P(z) / z$ as

$$
P_{1}(z)=\frac{P(z)}{z}=\frac{a_{0}}{z}(1+\psi(z))
$$

where $\psi(z)$ is holomorphic in $\mathbb{C}$ and $|\psi(z)| \leq 1 / 4$ if $|z| \leq R_{2}$. For each $z_{0} \in$ $\overline{\mathbb{D}}\left(0, R_{2} / 2\right) \backslash\{0\}$ and $z \in \partial \mathbb{D}\left(0, R_{2}\right)$, we have

$$
\left|z-z_{0}\right|>\frac{1}{4}\left(|z|+\left|z_{0}\right|\right)
$$

Hence

$$
\left|\frac{1}{z}-\frac{1}{z_{0}}\right|>\frac{1}{4} \frac{|z|+\left|z_{0}\right|}{\left|z z_{0}\right|} \geq\left|\frac{\psi(z)}{z}-\frac{\psi\left(z_{0}\right)}{z_{0}}\right| .
$$

Define $\psi_{1}(z):=a_{0}\left(1 / z-1 / z_{0}\right)$ and $\psi_{2}(z):=P_{1}(z)-P_{1}\left(z_{0}\right)=\frac{a_{0}}{z}(1+\psi(z))-\frac{a_{0}}{z_{0}}(1+$ $\left.\psi\left(z_{0}\right)\right)$. By Rouché's theorem, $\psi_{1}(z)=0$ and $\psi_{2}(z)=0$ have the same number of roots in $\mathbb{D}\left(0, R_{2}\right)$. Since $\psi_{1}(z)=0$ has exactly one root $z_{0}$ in $\mathbb{D}\left(0, R_{2}\right)$, this means that $\psi_{2}(z)=0$ has exactly one root in $\mathbb{D}\left(0, R_{2}\right)$. By the arbitrariness of $z_{0}$, it means that $P_{1}(z)=P(z) / z$ is univalent in a neighborhood of $\overline{\mathbb{D}}\left(0, R_{2} / 2\right)$. 
Since $P$ is a polynomial, it is easy to see that $P\left(e^{z}\right) / e^{z}$ is a transcendental entire function. We now give some quantitative estimations on the mapping properties of $f(z)=P\left(e^{z}\right) / e^{z}$ by applying some properties of $P(z) / z$ obtained above. Recall that $\Lambda(x)=\{z \in \mathbb{C}:|\operatorname{Re} z|>x\}$ for $x>0$. We denote

$$
K_{0}:=\min \left\{\left|a_{0}\right|,\left|a_{N}\right|\right\}>0 .
$$

Corollary 2.7. Let

$$
r_{0}:=\frac{\pi}{N-1} \quad \text { and } \quad R_{3}:=\log \left(2+\frac{8 K}{K_{0}}\right) .
$$

Then for any square $Q \subset \Lambda\left(R_{3}\right)$ with sides of length $r \leq r_{0}$, the restriction of $f(z)=P\left(e^{z}\right) / e^{z}$ on a neighbourhood of $Q$ is a conformal map.

Proof. We have $\left|e^{z}\right| \geq 2 R_{1}$ if $\operatorname{Re} z \geq \log \left(2 R_{1}\right)$ and $\left|e^{z}\right| \leq R_{2} / 2$ if $\operatorname{Re} z \leq \log \left(R_{2} / 2\right)$. Let $Q \subset \Lambda\left(R_{3}\right)$ be a square with sides of length $\pi /(N-1)$. It is easy to see that $\exp$ is injective in a neighbourhood of $Q$ and $\exp (Q)$ is contained in $\overline{\mathbb{D}}\left(0, R_{2} / 2\right)$ or $\mathbb{U}\left(2 R_{1}, \theta, \frac{\pi}{N-1}\right)$ for some $\theta \in[0,2 \pi)$. This means that $f(z)=P\left(e^{z}\right) / e^{z}$ is conformal in a neighborhood of $Q$ by Lemma 2.6.

We will use the following lemma to estimate $\left|f^{\prime}(z)\right|$ and $\left|f^{\prime \prime}(z) / f^{\prime}(z)\right|$ for $f(z)=$ $P\left(e^{z}\right) / e^{z}$.

Lemma 2.8. Suppose that $|z| \geq R_{4}$ or $|z| \leq R_{5}$, where

$$
R_{4}=1+\max \left\{\frac{2 K+4}{\left|a_{N}\right|}, \frac{K}{\left|a_{N}\right|}\left(\frac{2 N^{2}}{N-1}+1\right)\right\} \text { and } R_{5}=\min \left\{\frac{\left|a_{0}\right|}{2(K N+2)}, \frac{1}{2 N} \sqrt{\frac{\left|a_{0}\right|}{K}}\right\} .
$$

Then

$$
\left|P^{\prime}(z)-\frac{P(z)}{z}\right|>2 \text { and } \quad\left|\frac{z^{2} P^{\prime \prime}(z)}{z P^{\prime}(z)-P(z)}-1\right|<N .
$$

Proof. A direct calculation shows that

$$
P^{\prime}(z)=\sum_{k=1}^{N} k a_{k} z^{k-1} \quad \text { and } \quad P^{\prime \prime}(z)=\sum_{k=2}^{N} k(k-1) a_{k} z^{k-2} .
$$

This means that

$$
P^{\prime}(z)-\frac{P(z)}{z}=\sum_{k=1}^{N} k a_{k} z^{k-1}-\sum_{k=0}^{N} a_{k} z^{k-1}=\sum_{k=0}^{N}(k-1) a_{k} z^{k-1}
$$

and

$$
\frac{z^{2} P^{\prime \prime}(z)}{z P^{\prime}(z)-P(z)}-1=\frac{\sum_{k=0}^{N} k(k-1) a_{k} z^{k}}{\sum_{k=0}^{N}(k-1) a_{k} z^{k}}-1=\frac{\sum_{k=0}^{N}(k-1)^{2} a_{k} z^{k}}{\sum_{k=0}^{N}(k-1) a_{k} z^{k}} .
$$

If $|z| \geq 1+\frac{2 K+4}{\left|a_{N}\right|}>3$, by 17 we have

$$
\begin{aligned}
\left|P^{\prime}(z)-\frac{P(z)}{z}\right| & \geq\left|a_{N}\right|(N-1)|z|^{N-1}-K(N-1)\left(|z|^{N-2}+\cdots+|z|+1\right) \\
& \geq(N-1)|z|^{N-1}\left(\left|a_{N}\right|-\frac{K}{|z|-1}\right) \\
& \geq \frac{\left|a_{N}\right|}{2}|z|^{N-1} \geq \frac{\left|a_{N}\right|}{2}|z|>2 .
\end{aligned}
$$

If $|z| \leq \frac{\left|a_{0}\right|}{2(K N+2)}<\frac{1}{2}$, we have

$$
\begin{aligned}
\left|P^{\prime}(z)-\frac{P(z)}{z}\right| & \geq \frac{\left|a_{0}\right|}{|z|}-K(N-1)\left(|z|+\cdots+|z|^{N-1}\right) \\
& \geq \frac{\left|a_{0}\right|}{|z|}-K(N-1)>\frac{\left|a_{0}\right|}{2|z|} \geq K N+2>2 .
\end{aligned}
$$


For the second inequality, if $|z| \geq 1+\frac{K}{\left|a_{N}\right|}\left(\frac{2 N^{2}}{N-1}+1\right)>8$, by 18 we have

$$
\begin{aligned}
\left|\frac{z^{2} P^{\prime \prime}(z)}{z P^{\prime}(z)-P(z)}-1\right| & \leq N-1+\left|\frac{\sum_{k=0}^{N-1}(k-1)(N-k) a_{k} z^{k}}{\sum_{k=0}^{N}(k-1) a_{k} z^{k}}\right| \\
& \leq N-1+\frac{K N^{2}}{N-1} \cdot \frac{|z|+\cdots+|z|^{N-1}}{\left|a_{N}\right||z|^{N}-K\left(|z|+\cdots+|z|^{N-1}\right)} \\
& \leq N-1+\frac{K N^{2}}{N-1} \cdot \frac{1}{\left|a_{N}\right|(|z|-1)-K} \leq N-\frac{1}{2}<N .
\end{aligned}
$$

If $|z| \leq \frac{1}{2 N} \sqrt{\left|a_{0}\right| / K}<\frac{1}{2}$, by $(18)$ we have

$$
\begin{aligned}
\left|\frac{z^{2} P^{\prime \prime}(z)}{z P^{\prime}(z)-P(z)}-1\right| & \leq 1+\left|\frac{\sum_{k=2}^{N} k(k-1) a_{k} z^{k}}{\sum_{k=0}^{N}(k-1) a_{k} z^{k}}\right| \\
& \leq 1+\frac{K N^{2}\left(|z|^{2}+\cdots+|z|^{N}\right)}{\left|a_{0}\right|-K N\left(|z|^{2}+\cdots+|z|^{N}\right)} \\
& \leq 1+\frac{2 K N^{2}|z|^{2}}{\left|a_{0}\right|-2 K N|z|^{2}} \leq 1+\frac{N}{2 N-1} \leq \frac{5}{3}<N
\end{aligned}
$$

Corollary 2.9. Let

$$
R_{6}:=\max \left\{\log R_{4},-\log R_{5}\right\} .
$$

Then for any $z \in \Lambda\left(R_{6}\right)$, the function $f(z)=P\left(e^{z}\right) / e^{z}$ satisfies

$$
\left|f^{\prime}(z)\right|>2 \text { and } \frac{\left|f^{\prime \prime}(z)\right|}{\left|f^{\prime}(z)\right|}<N .
$$

Proof. Denote $P_{1}(w):=P(w) / w$. Therefore, $f(z)=P\left(e^{z}\right) / e^{z}=P_{1} \circ \exp (z)$. It is easy to check that

$$
f^{\prime}(z)=P_{1}^{\prime}\left(e^{z}\right) e^{z} \quad \text { and } \quad f^{\prime \prime}(z)=P_{1}^{\prime \prime}\left(e^{z}\right) e^{2 z}+P_{1}^{\prime}\left(e^{z}\right) e^{z}
$$

Let $w=e^{z}$. By a straightforward computation, we have

$$
f^{\prime}(z)=P_{1}^{\prime}(w) w=P^{\prime}(w)-\frac{P(w)}{w}
$$

and

$$
\frac{f^{\prime \prime}(z)}{f^{\prime}(z)}=\frac{P_{1}^{\prime \prime}(w) w^{2}+P_{1}^{\prime}(w) w}{P_{1}^{\prime}(w) w}=\frac{w^{2} P^{\prime \prime}(w)}{w P^{\prime}(w)-P(w)}-1 .
$$

Then the result follows from Lemma 2.8 immediately.

2.4. Escaping and fast escaping sets. Let $f$ be a transcendental entire function. A point $a \in \mathbb{C}$ is called an asymptotic value of $f$ if there exists a continuous curve $\gamma(t) \subset \mathbb{C}$ with $0<t<\infty$, such that $\gamma(t) \rightarrow \infty$ as $t \rightarrow \infty$ and $f(\gamma(t)) \rightarrow a$ as $t \rightarrow \infty$.

Lemma 2.10. The entire function $f(z)=P\left(e^{z}\right) / e^{z}$ does not have any finite asymptotic value.

Proof. Assume that $a \in \mathbb{C}$ is a finite asymptotic value of $f(z)$. Then by definition, there exists a continuous curve $\gamma(t) \subset \mathbb{C}$ with $0<t<\infty$, such that $\gamma(t) \rightarrow \infty$ as $t \rightarrow \infty$ and $f(\gamma(t)) \rightarrow a$ as $t \rightarrow \infty$. This means that

$$
\lim _{t \rightarrow \infty} \frac{P(w)}{w} \circ e^{\gamma(t)}=a
$$

Denote $\gamma(t)=x(t)+\mathrm{i} y(t)$ and let $w_{1}, w_{2}, \cdots, w_{N}$ be the $N$ roots of the equation $P(w)=a w$. We define the set $Y:=\left\{\arg w_{i}+2 k \pi: 1 \leq i \leq N, k \in \mathbb{Z}\right\}$. If $x(t)$ is unbounded as $t \rightarrow \infty$, then $f(\gamma(t))$ is also unbounded and this is a contradiction. Hence $|x(t)| \leq A$ for some constant $A>0$ for all $t$. Since $\gamma(t) \rightarrow \infty$ 
as $t \rightarrow \infty$, this implies that $y(t) \rightarrow \infty$ as $t \rightarrow \infty$. Therefore, for each $y_{0} \in$ $\mathbb{R} \backslash Y$, there exists a sequence $\left\{z_{n}\right\} \subset \gamma(t)$ such that $\operatorname{Im} z_{n} \rightarrow \infty$ as $n \rightarrow \infty$ and $\lim _{n \rightarrow \infty} e^{\mathrm{i} I m z_{n}}=e^{\mathrm{i} y_{0}}$. Since $|x(t)| \leq A$, it follows that $\lim _{t \rightarrow \infty} e^{x(t)} \neq 0$. This implies that $\lim _{n \rightarrow \infty} f\left(z_{n}\right)=\lim _{n \rightarrow \infty} P\left(e^{z_{n}}\right) / e^{z_{n}} \neq a$, which is a contradiction.

Let $f$ be a transcendental entire function. The set

$$
I(f):=\left\{z \in \mathbb{C}: f^{\circ n}(z) \rightarrow \infty \text { as } n \rightarrow \infty\right\}
$$

is called the escaping set of $f$. We use $\operatorname{sing}\left(f^{-1}\right)$ to denote the set of singular values of $f$ which consists of all the critical values and asymptotic values of $f$ and their accumulation points.

Corollary 2.11. The escaping set $I(f)$ of $f(z)=P\left(e^{z}\right) / e^{z}$ is contained in the Julia set $J(f)$.

Proof. It is clear that the set of the critical values of $f(z)=P\left(e^{z}\right) / e^{z}$ is finite. From Lemma 2.10, it follows that $\operatorname{sing}\left(f^{-1}\right)$ is bounded. According to EL92, Theorem $1]$, we have $I(f) \subset J(f)$.

Actually, we will estimate the area of the complement of the fast escaping set in next section. Let $f$ be a transcendental entire function. The maximal modulus function is defined by

$$
M(r, f):=\max _{|z|=r}|f(z)|, \text { where } r>0 .
$$

We use $M^{\circ n}(r, f)$ to denote the $n$-th iterate of $M(r, f)$ with respect to the variable $r>0$, where $n \in \mathbb{N}$. The notation $M(r, f)$ is written as $M(r)$ if the function $f$ is known clearly. A subset of the escaping set, called the fast escaping set $A(f)$ was introduced in [BH99] and can be defined [RS12] by

$$
A(f):=\left\{z: \text { there is } \ell \in \mathbb{N} \text { such that }\left|f^{\circ(n+\ell)}(z)\right| \geq M^{\circ n}(R) \text { for } n \in \mathbb{N}\right\} .
$$

Here $R>0$ is a constant such that $M^{\circ n}(R) \rightarrow \infty$ as $n \rightarrow \infty$. It is proved in RS12, Theorem 2.2(b)] that $A(f)$ is independent of the choice of $R$ such that $M^{\circ n}(R) \rightarrow \infty$ as $n \rightarrow \infty$.

Lemma 2.12. Let $R>0$ be a constant and define $u_{0}:=R$. For $n \geq 1$, define $u_{n}$ inductively by $u_{n}:=R e^{R u_{n-1}}$. Let $v_{0} \in \mathbb{R}$ and define $v_{n}$ inductively by $v_{n}:=e^{v_{n-1}}$ for $n \geq 1$. Then there is $\ell \in \mathbb{N}$ such that $v_{n+\ell} \geq 2 R u_{n}$ for all $n \in \mathbb{N}$.

Proof. For any $v_{0} \in \mathbb{R}$, there exists an integer $\ell \in \mathbb{N}$ such that $v_{\ell} \geq 2 R^{2}$. Shifting the subscript of $\left(v_{n}\right)_{n \in \mathbb{N}}$ if necessary, it is sufficient to prove that if $v_{0} \geq 2 R^{2}$, then $v_{n} \geq 2 R u_{n}$ for all $n \in \mathbb{N}$. Suppose that $v_{n-1} \geq 2 R u_{n-1}$ for some $n \geq 1$ (note that $\left.v_{0} \geq 2 R u_{0}\right)$. We hope to obtain that $v_{n} \geq 2 R u_{n}$. Note that $v_{n}=e^{v_{n-1}} \geq e^{2 R u_{n-1}}$ and $u_{n}=R e^{R u_{n-1}}$. It is sufficient to obtain $R u_{n-1} \geq \log \left(2 R^{2}\right)$. This is true since $u_{n-1} \geq R$ and $R^{2} \geq \log \left(2 R^{2}\right)$ for all $R>0$.

Corollary 2.13. Let $z_{0} \in \mathbb{C}$ and suppose that $z_{n}=f^{\circ n}\left(z_{0}\right)$ satisfies $\left|z_{n}\right| \geq \xi_{n}$ for all $n \in \mathbb{N}$, where $\xi_{n}>0$ is defined inductively by

$$
\xi_{n}=2 \exp \left(\xi_{n-1} / 2\right) \text { with } \xi_{0}>0 .
$$

Then $z_{0}$ is contained in the fast escaping set of $f(z)=P\left(e^{z}\right) / e^{z}$.

Proof. Recall that $N \geq 2$ is the degree of the polynomial $P$ and $K>0$ is defined in (12). According to Lemma 2.5, there exists $\delta_{0} \geq 1$ such that if $\delta \geq \delta_{0}$, then the maximal modulus function of $f$ satisfies

$$
M(\delta)=M(\delta, f) \leq 2 K e^{(N-1) \delta} .
$$


On the other hand, there exists $\delta_{1}>0$ such that for all $\delta \geq \delta_{1}$, then $M^{\circ n}(\delta)$ is monotonically increasing as $n$ increases. Since the Julia set of $f$ is non-empty, this means that $M^{\circ n}(\delta) \rightarrow \infty$ as $n \rightarrow \infty$ if $\delta \geq \delta_{1}$.

Define

$$
R:=\max \left\{2 K,(N-1) \delta_{0}, \delta_{1}\right\} \geq 1 .
$$

We denote $u_{0}=R$ and for $n \geq 1$, define $u_{n}$ inductively by $u_{n}=R e^{R u_{n-1}}$. Then we have $M^{\text {on }}(R) \leq u_{n}$ for all $n \in \mathbb{N}$. By the definition of $\xi_{n}$, we have $\xi_{n}=$ $2 \exp ^{\circ n}\left(\xi_{0} / 2\right)$. Let $v_{0}:=\xi_{0} / 2$ and define $v_{n}:=e^{v_{n-1}}$ for $n \geq 1$. According to Lemma 2.12, there exists $\ell \in \mathbb{N}$ such that for all $n \in \mathbb{N}$,

$$
\left|f^{\circ(n+\ell)}\left(z_{0}\right)\right|=\left|z_{n+\ell}\right| \geq \xi_{n+\ell}=2 v_{n+\ell} \geq 4 R u_{n} \geq u_{n} \geq M^{\circ n}(R) .
$$

By the defintion of $R$, we have $M^{\circ n}(R) \rightarrow \infty$ as $n \rightarrow \infty$. This means that $z_{0}$ is contained in the fast escaping set of $f$.

\section{Proof of the theorems}

3.1. Proof of Theorem 1.1. Recall that $N \geq 2$ is the degree of the polynomial $P$. Let $r>0$ be fixed such that

$$
r \leq \frac{1}{4 N}
$$

We define

$$
x^{\prime}:=\max \left\{R_{3}, R_{6}, 6 \log 2\right\},
$$

where $R_{3}$ and $R_{6}$ are constants introduced in Corollary 2.7 and Corollary 2.9 respectively.

Recall that $\Lambda(x)=\{z \in \mathbb{C}:|\operatorname{Re} z|>x\}$ is the set defined in $\sqrt{5}$ for all $x>0$. Let $Q_{0}$ be a square in $\Lambda(x)$ with sides of length $r$, where $x \geq x^{\prime}$. Since $r<r_{0}=$ $\pi /(N-1)$, from Corollary 2.7 we know that $f$ is conformal in a neighbourhood of $Q_{0}$. For $k \in \mathbb{N}$, define

$$
x_{k}:=2 \exp ^{\circ k}(x / 2)
$$

In particular, $x_{0}=x \geq x^{\prime}$ and we have $x_{k+1}=2 \exp \left(x_{k} / 2\right)>x_{k} \geq x^{\prime}$ since $2 e^{x / 2}>x$ for all $x \in \mathbb{R}$. Recall that $\mathcal{Q}_{r}$ is a collection of grids with sides of length $r>0$ defined in (6). For any subset $E$ of $Q_{0}$ in $\Lambda\left(x_{0}\right)$ and $k \in \mathbb{N}$, define

$$
\operatorname{pack}\left(f^{\circ k}(E)\right):=\left\{Q_{r} \in \mathcal{Q}_{r}: Q_{r} \subset f^{\circ k}(E) \cap \Lambda\left(x_{k}\right)\right\} .
$$

We now define a sequence of families of measurable sets satisfying the nesting conditions based on the square $Q_{0}$. Let $\mathcal{E}_{0}:=\left\{Q_{0}\right\}$ and for $k \geq 1$, define inductively

$$
\mathcal{E}_{k}:=\left\{F_{k} \subset Q_{0}: F_{k} \subset E_{k-1} \in \mathcal{E}_{k-1} \text { and } f^{\circ k}\left(F_{k}\right) \in \operatorname{pack}\left(f^{\circ k}\left(E_{k-1}\right)\right)\right\} .
$$

It is clear that $\mathcal{E}_{k}$ is a finite collection of measurable subsets of $\mathbb{C}$ for all $k \in \mathbb{N}$. Denote the elements of $\mathcal{E}_{k}$ by $E_{k, i}$, where $1 \leq i \leq d_{k}$.

By definition, for all $k \in \mathbb{N}$, we have $f^{\circ(k+1)}\left(E_{k, i}\right)=f\left(Q_{r}^{k}\right)$, wherf ${ }^{3} Q_{r}^{k}$ is a square with sides of length $r$ and $Q_{r}^{k} \subset \Lambda\left(x_{k}\right)$. From (4), Corollary 2.9 and (25), we have

$$
N\left(\left.f\right|_{Q_{r}^{k}}\right)<N \sqrt{2} r \leq \frac{\sqrt{2}}{4}
$$

By Lemma 2.1, the distortion of $f$ on $Q_{r}^{k}$ satisfies

$$
L\left(\left.f\right|_{Q_{r}^{k}}\right) \leq 1+2 N\left(\left.f\right|_{Q_{r}^{k}}\right)<2 .
$$

\footnotetext{
${ }^{3}$ Note that $Q_{r}^{k} \subset \Lambda\left(x_{k}\right)$ is a square depending also on the subscript ' $i$ ' of $E_{k, i}$, where $k \in \mathbb{N}$ and $1 \leq i \leq d_{k}$. We omit this index here for simplicity.
} 
For every $k \in \mathbb{N}$, let $z_{k}$ be any point in $Q_{r}^{k} \subset \Lambda\left(x_{k}\right)$. From (2) and 28) we have

$$
\begin{aligned}
\operatorname{Area}\left(f\left(Q_{r}^{k}\right)\right) & =\int_{Q_{r}^{k}}\left|f^{\prime}(z)\right|^{2} d x d y \geq \inf _{z \in Q_{r}^{k}}\left|f^{\prime}(z)\right|^{2} \cdot \operatorname{Area}\left(Q_{r}^{k}\right) \\
& \geq \frac{\left|f^{\prime}\left(z_{k}\right)\right|^{2}}{\left(L\left(\left.f\right|_{Q_{r}^{k}}\right)\right)^{2}} \cdot r^{2}>\frac{1}{4}\left|f^{\prime}\left(z_{k}\right)\right|^{2} r^{2}
\end{aligned}
$$

and

$$
\begin{aligned}
\operatorname{diam}\left(f\left(Q_{r}^{k}\right)\right) & \leq \sup _{z \in Q_{r}^{k}}\left|f^{\prime}(z)\right| \cdot \operatorname{diam}\left(Q_{r}^{k}\right) \\
& \leq L\left(\left.f\right|_{Q_{r}^{k}}\right)\left|f^{\prime}\left(z_{k}\right)\right| \cdot \sqrt{2} r<2 \sqrt{2}\left|f^{\prime}\left(z_{k}\right)\right| r .
\end{aligned}
$$

Recall that $K_{0}=\min \left\{\left|a_{0}\right|,\left|a_{N}\right|\right\}>0$ is the constant defined in (15). By (19), 20 and $(22)$, we have

$$
\left|f^{\prime}\left(z_{k}\right)\right|>\frac{1}{2} K_{0} e^{\left|\operatorname{Re} z_{k}\right|}>\frac{1}{2} K_{0} e^{x_{k}}
$$

For $k \in \mathbb{N}$ and $1 \leq i \leq d_{k}$, we denote

$$
B_{1}:=\cup\left\{Q_{r} \in \mathcal{Q}_{r}: Q_{r} \subset f^{\circ(k+1)}\left(E_{k, i}\right) \cap\left(\mathbb{C} \backslash \Lambda\left(x_{k+1}\right)\right)\right\}
$$

and

$$
B_{2}:=\cup\left\{Q_{r} \in \mathcal{Q}_{r}: Q_{r} \cap\left(\partial f^{\circ(k+1)}\left(E_{k, i}\right) \cup\left(\partial \Lambda\left(x_{k+1}\right) \cap f^{\circ(k+1)}\left(E_{k, i}\right)\right)\right) \neq \emptyset\right\} .
$$

Recall that $f^{\circ(k+1)}\left(E_{k, i}\right)=f\left(Q_{r}^{k}\right)$ for some square $Q_{r}^{k}$ in $\Lambda\left(x_{k}\right)$ with sides of length $r$, where $k \in \mathbb{N}$ and $1 \leq i \leq d_{k}$. From (29), (30) and (31), we have

$$
\begin{aligned}
& \frac{\operatorname{Area}\left(B_{1}\right)}{\operatorname{Area}\left(f^{\circ(k+1)}\left(E_{k, i}\right)\right)} \leq \frac{2 x_{k+1} \operatorname{diam}\left(f^{\circ(k+1)}\left(E_{k, i}\right)\right)}{\operatorname{Area}\left(f^{\circ}(k+1)\left(E_{k, i}\right)\right)} \\
= & \frac{2 x_{k+1} \operatorname{diam}\left(f\left(Q_{r}^{k}\right)\right)}{\operatorname{Area}\left(f\left(Q_{r}^{k}\right)\right)}<\frac{16 \sqrt{2} x_{k+1}}{\left|f^{\prime}\left(z_{k}\right)\right| r}<\frac{32 \sqrt{2}}{K_{0} r} \cdot \frac{x_{k+1}}{e^{x_{k}}} .
\end{aligned}
$$

Note that $x_{k+1} \geq x_{1}=2 e^{x / 2}$ for all $k \in \mathbb{N}$ and $x \geq 6 \log 2$ by 26. By Lemma 2.4. 28, 29 and (31), we have

$$
\begin{aligned}
& \frac{\operatorname{Area}\left(B_{2}\right)}{\operatorname{Area}\left(f^{k+1}\left(E_{k, i}\right)\right)} \leq \frac{\left(16+12 \sqrt{2} L\left(\left.f\right|_{Q_{r}^{k}}\right)\left|f^{\prime}\left(z_{k}\right)\right|\right) r^{2}}{\operatorname{Area}\left(f\left(Q_{r}^{k}\right)\right)} \\
< & \frac{32\left(2+3 \sqrt{2}\left|f^{\prime}\left(z_{k}\right)\right|\right)}{\left|f^{\prime}\left(z_{k}\right)\right|^{2}}<\frac{256}{K_{0}^{2} e^{2 x_{k}}}+\frac{192 \sqrt{2}}{K_{0} e^{x_{k}}} \\
\leq & \left(\frac{128}{K_{0}^{2}} \cdot \frac{1}{e^{3 x / 2}}+\frac{96 \sqrt{2}}{K_{0}} \cdot \frac{1}{e^{x / 2}}\right) \cdot \frac{x_{k+1}}{e^{x_{k}}} \leq\left(\frac{1}{4 K_{0}^{2}}+\frac{12 \sqrt{2}}{K_{0}}\right) \cdot \frac{x_{k+1}}{e^{x_{k}}} .
\end{aligned}
$$

For all $k \in \mathbb{N}$ and $1 \leq i \leq d_{k}$, by $(32)$ and 33 , we have

$$
\begin{aligned}
& \operatorname{density}\left(\bigcup \operatorname{pack}\left(f^{\circ(k+1)}\left(E_{k, i}\right)\right), f^{\circ(k+1)}\left(E_{k, i}\right)\right) \\
\geq & \frac{\operatorname{Area}\left(\bigcup\left\{Q_{r} \in \mathcal{Q}_{r}: Q_{r} \cap f^{\circ(k+1)}\left(E_{k, i}\right) \neq \emptyset\right)\right.}{\operatorname{Area}\left(f^{\circ(k+1)}\left(E_{k, i}\right)\right)}-\frac{\operatorname{Area}\left(B_{1}\right)+\operatorname{Area}\left(B_{2}\right)}{\operatorname{Area}\left(f^{\circ(k+1)}\left(E_{k, i}\right)\right)} \\
> & 1-c_{0} \frac{x_{k+1}}{e^{x_{k}}} \geq 1-c_{1} \frac{x_{k+1}}{e^{x_{k}}},
\end{aligned}
$$

where

$$
c_{1} \geq c_{0}:=\frac{32 \sqrt{2}}{K_{0} r}+\frac{1}{4 K_{0}^{2}}+\frac{12 \sqrt{2}}{K_{0}} .
$$

Comparing (26), we assume that $x^{*}>0$ is a fixed constant such that

$$
x^{*} \geq \max \left\{R_{3}, R_{6}, 6 \log 2,12+2 \log c_{1}\right\} .
$$


Moreover, we suppose that the sequence $\left\{x_{k}\right\}_{k \in \mathbb{N}}$ in 27 is chosen such that the initial point satisfies $x_{0}=x \geq x^{*}$. Then, all the statements above are still true since $x^{*} \geq x^{\prime}$.

By a straightforward induction, one can show that for all $k \in \mathbb{N}$ and $x \in \mathbb{R}$,

$$
\exp ^{\circ(k+1)}(x) \geq \exp (k) \exp (x) .
$$

Since $x_{k+1}=2 e^{x_{k} / 2}$, we have

$$
\frac{x_{k+1}}{e^{x_{k}}}=\frac{2}{e^{x_{k} / 2}}=\frac{2}{\exp ^{\circ(k+1)}(x / 2)} \leq \frac{2}{e^{k}} \cdot \frac{1}{e^{x / 2}} .
$$

On the other hand, by (36), we have $e^{x / 2} \geq c_{1} e^{6}>6 c_{1} e^{4}$ since $x \geq x_{*}$. Therefore,

$$
c_{1} e^{4} \frac{x_{k+1}}{e^{x_{k}}} \leq c_{1} e^{4} \cdot \frac{2}{e^{k}} \cdot \frac{1}{e^{x / 2}} \leq c_{1} e^{4} \cdot \frac{2}{e^{x / 2}}<\frac{1}{3} .
$$

Define $V:=f\left(Q_{r}^{k}\right)$ and let $G:=f^{-(k+1)}: V \rightarrow Q_{0}$ be the inverse of $\left.f^{\circ(k+1)}\right|_{E_{k, i}}$, where $k \in \mathbb{N}$ and $1 \leq i \leq d_{k}$. By Lemma 2.2. Corollary 2.9 and (25), the distortion of $G$ on $V$ satisfies

$$
L\left(\left.G\right|_{V}\right)<\exp \left(\frac{2}{2-1}\right)=e^{2} .
$$

From (3) and (39), we have

$$
\begin{aligned}
& \operatorname{density}\left(\mathcal{E}_{k+1}, E_{k, i}\right)=1-\operatorname{density}\left(E_{k, i} \backslash \mathcal{E}_{k+1}, E_{k, i}\right) \\
= & 1-\operatorname{density}\left(G\left(f^{\circ(k+1)}\left(E_{k, i} \backslash \mathcal{E}_{k+1}\right)\right), G\left(f^{\circ(k+1)}\left(E_{k, i}\right)\right)\right) \\
\geq & 1-L\left(\left.G\right|_{V}\right)^{2} \operatorname{density}\left(f^{\circ(k+1)}\left(E_{k, i}\right) \backslash \bigcup \operatorname{pack}\left(f^{\circ(k+1)}\left(E_{k, i}\right), f^{\circ(k+1)}\left(E_{k, i}\right)\right)\right. \\
\geq & 1-e^{4}\left(1-\operatorname{density}\left(\bigcup \operatorname{pack}\left(f^{\circ(k+1)}\left(E_{k, i}\right)\right), f^{\circ(k+1)}\left(E_{k, i}\right)\right)\right) .
\end{aligned}
$$

Therefore, by (34) and (38), we have

$$
\operatorname{density}\left(\mathcal{E}_{k+1}, E_{k, i}\right) \geq 1-c_{1} e^{4} \frac{x_{k+1}}{e^{x_{k}}} \geq \frac{2}{3},
$$

where $k \in \mathbb{N}$ and $1 \leq i \leq d_{k}$. For all $k \in \mathbb{N}$, by setting

$$
\rho_{k}:=1-c_{1} e^{4} \frac{x_{k+1}}{e^{x_{k}}},
$$

it is easy to see that $\left\{\mathcal{E}_{k}\right\}_{k=0}^{\infty}$ satisfies the nesting conditions.

Define $E=\cap_{k=0}^{\infty} \mathcal{E}_{k}$. Recall that $A(f)$ is the fast escaping set of $f$ defined in (24). Since every point $z \in E_{k, i}$ satisfies $f^{\circ j}(z) \in \Lambda\left(x_{j}\right)$ for $0 \leq j \leq k$ and $x_{k} \rightarrow+\infty$ as $k \rightarrow \infty$, it means that $E$ is contained in the fast escaping set $A(f)$ by (27) and Corollary 2.13. According to Lemma 2.3, we have

$$
\operatorname{density}\left(A(f), Q_{0}\right) \geq \operatorname{density}\left(E, Q_{0}\right) \geq \prod_{k=0}^{\infty} \rho_{k} .
$$

Note that $\log (1-t)>-2 t$ for $t \in(0,1 / 2)$. By 38 and 41 we have

$$
\begin{aligned}
\log \left(\prod_{k=0}^{\infty} \rho_{k}\right) & =\sum_{k=0}^{\infty} \log \left(1-c_{1} e^{4} \frac{x_{k+1}}{e^{x_{k}}}\right) \geq-2 \sum_{k=0}^{\infty} c_{1} e^{4} \frac{x_{k+1}}{e^{x_{k}}} \\
& \geq-\frac{4 c_{1} e^{4}}{e^{x / 2}} \sum_{k=0}^{\infty} \frac{1}{e^{k}}>-\frac{8 c_{1} e^{4}}{e^{x / 2}} .
\end{aligned}
$$

Since $e^{-t} \geq 1-t$ for all $t \in \mathbb{R}$, we have

$$
\operatorname{density}\left(A(f), Q_{0}\right)>\exp \left(-\frac{8 c_{1} e^{4}}{e^{x / 2}}\right) \geq 1-\frac{8 c_{1} e^{4}}{e^{x / 2}}
$$

for all $x \geq x^{*}$ and all square $Q_{0} \subset \Lambda(x)$ with sides of length $r$. 
Theorem 3.1. Let $S$ be any horizontal strip of width $2 \pi$. Then the area of the complement of the fast escaping set of $f(z)=P\left(e^{z}\right) / e^{z}$ satisfies

$$
\operatorname{Area}\left(S \cap A(f)^{c}\right) \leq(4 \pi+4 r)\left(x^{*}+r+8 c_{1} e^{4-x^{*} / 2} \frac{r}{1-e^{-r / 2}}\right)<\infty,
$$

where $r, c_{1}$ and $x^{*}$ are any positive constants satisfying (25), (35) and (36) respectively.

Proof. Define the half strip $S_{+}$by

$$
S_{+}:=\{z \in \mathbb{C}: 0 \leq \operatorname{Im} z \leq 2 \pi \text { and } \operatorname{Re} z \geq 0\} .
$$

We take

$$
m_{0}=\left[x^{*} / r\right]+1 \text { and } n_{0}=[2 \pi / r]+1
$$

where $[x]$ denotes the integer part of $x \geq 0$. Recall that $Q_{r}^{m, n}$ is defined as

$$
Q_{r}^{m, n}:=\{z \in \mathbb{C}: m r \leq \operatorname{Re} z \leq(m+1) r \text { and } n r \leq \operatorname{Im} z \leq(n+1) r\},
$$

where $m, n \in \mathbb{Z}$. Since $Q_{r}^{m, n} \subset \Lambda\left(x^{*}\right)$ for all $m \geq m_{0}$, we get

$$
\operatorname{density}\left(A(f), Q_{r}^{m, n}\right)>1-\frac{8 c_{1} e^{4}}{\exp (m r / 2)}
$$

for all $m \geq m_{0}$ by 42 . So

$$
\begin{aligned}
\operatorname{Area}\left(S_{+} \cap A(f)^{c}\right) & \leq \operatorname{Area}\left(\left(\bigcup_{m=0}^{\infty} \cup_{n=0}^{n_{0}} Q_{r}^{m, n}\right) \backslash A(f)\right) \\
& \leq \sum_{m=0}^{\infty} \sum_{n=0}^{n_{0}} \operatorname{Area}\left(Q_{r}^{m, n} \backslash A(f)\right) \\
& \leq \sum_{m=0}^{\infty} \sum_{n=0}^{n_{0}}\left(1-\operatorname{density}\left(A(f), Q_{r}^{m, n}\right)\right) \cdot \operatorname{Area}\left(Q_{r}^{m, n}\right) .
\end{aligned}
$$

By 44) and 45, we obtain

$$
\begin{aligned}
\operatorname{Area}\left(S_{+} \cap A(f)^{c}\right) & \leq r^{2}\left(\sum_{m=0}^{m_{0}-1} \sum_{n=0}^{n_{0}} 1+\sum_{m=m_{0}}^{\infty} \sum_{n=0}^{n_{0}} \frac{8 c_{1} e^{4}}{\exp (m r / 2)}\right) \\
& \leq(2 \pi+2 r)\left(x^{*}+r+8 c_{1} e^{4-x^{*} / 2} \frac{r}{1-e^{-r / 2}}\right)
\end{aligned}
$$

This means that $\operatorname{Area}\left(S_{+} \cap A(f)^{c}\right)<\infty$ for every fixed $r>0$ satisfying [25. Similarly, one can obtain

$$
\operatorname{Area}\left(S_{-} \cap A(f)^{c}\right) \leq(2 \pi+2 r)\left(x^{*}+r+8 c_{1} e^{4-x^{*} / 2} \frac{r}{1-e^{-r / 2}}\right),
$$

where $S_{-}=\{z \in \mathbb{C}: 0 \leq \operatorname{Im} z \leq 2 \pi$ and $\operatorname{Re} z \leq 0\}$. Since $f(z)=f(z+2 \pi \mathrm{i})$, for any horizontal strip $S$ of width $2 \pi$, we have

$$
\operatorname{Area}\left(S \cap A(f)^{c}\right) \leq(4 \pi+4 r)\left(x^{*}+r+8 c_{1} e^{4-x^{*} / 2} \frac{r}{1-e^{-r / 2}}\right) .
$$

This completes the proof of Theorem 3.1 and hence Theorem 1.1 
3.2. Proof of Theorem $\mathbf{1 . 2}$, Consider the quadratic polynomial

$$
P(z)=\frac{\alpha}{2} z^{2}+\mathrm{i} \beta z-\frac{\alpha}{2} \text {, where } \alpha \neq 0 \text { and } \beta \in \mathbb{C} \text {. }
$$

We then have

$$
f(z):=\frac{P\left(e^{z}\right)}{e^{z}}=\frac{\alpha}{2} e^{z}+\mathrm{i} \beta-\frac{\alpha}{2} e^{-z} .
$$

Note that $\alpha \sin (z+\beta)$ is conjugated by $z \mapsto \mathrm{i}(z+\beta)$ to $f(z)$, In order to prove Theorem 1.2 , it is sufficient to prove the corresponding statements on $f$.

Now we collect all the needing constants in the proof. Note that the degree of $P$ is $\operatorname{deg}(P)=N=2$. By (25) we fix the choice of $r>0$ by setting

$$
r=1 / 8 .
$$

By (15), we have $K_{0}=|\alpha| / 2$. From (35), we fix

$$
c_{1}=c_{0}=\frac{536 \sqrt{2}}{|\alpha|}+\frac{1}{|\alpha|^{2}} .
$$

By 16 , we have

$$
R_{3}=\log \left(2+\frac{16 K}{|\alpha|}\right), \text { where } K=\max \{|\alpha| / 2,|\beta|\} .
$$

According to Lemma 2.8, we have

$$
R_{4}=\max \left\{1+\frac{4(K+2)}{|\alpha|}, 1+\frac{18 K}{|\alpha|}\right\} \quad \text { and } \quad R_{5}=\min \left\{\frac{|\alpha|}{8(K+1)}, \frac{1}{4} \sqrt{\frac{|\alpha|}{2 K}}\right\} .
$$

Since $K \geq|\alpha| / 2>0$, we have

$$
\frac{8(K+1)}{|\alpha|}>\frac{8 K}{|\alpha|} \geq 4 \sqrt{\frac{2 K}{|\alpha|}}, \quad \frac{8(K+1)}{|\alpha|}=\frac{4 K}{|\alpha|}+\frac{4(K+2)}{|\alpha|}>1+\frac{4(K+2)}{|\alpha|}
$$

and

Hence by $(36)$, we can fix

$$
1+\frac{18 K}{|\alpha|}=1+\frac{16 K}{|\alpha|}+\frac{K}{|\alpha| / 2} \geq 2+\frac{16 K}{|\alpha|} .
$$

$$
x^{*}=\max \left\{\log \left(1+\frac{18 K}{|\alpha|}\right), \log \left(\frac{8(K+1)}{|\alpha|}\right), 6 \log 2,12+2 \log c_{1}\right\} .
$$

By Theorem 3.1, the proof of Theorem 1.2 is finished module the statement on the sine and cosine functions.

Let $S$ be a vertical strip with width $2 \pi$. If $\alpha=1$ and $\beta=0$, then $K=1 / 2$ and

$$
r=1 / 8, \quad c_{1}=536 \sqrt{2}+1 \quad \text { and } \quad x^{*}=12+2 \log (536 \sqrt{2}+1) .
$$

From 43 we have

$$
\begin{aligned}
& \operatorname{Area}\left(S \cap A(\sin z)^{c}\right) \\
\leq & \left(4 \pi+\frac{1}{2}\right)\left(\frac{97}{8}+2 \log (536 \sqrt{2}+1)+\frac{1}{e^{2}-e^{31 / 16}}\right)<361 .
\end{aligned}
$$

If $\alpha=1$ and $\beta=\pi / 2$, then $K=\pi / 2$ and we still have 46. Also from 43. we have

$$
\text { Area }\left(S \cap A(\cos z)^{c}\right)<361 \text {. }
$$

This finishes the proof of Theorem 1.2 . 


\section{REFERENCES}

[AB12] M. Aspenberg and W. Bergweiler, Entire functions with Julia sets of positive measure, Math. Ann. 352 (2012), no. 1, 27-54.

[Bea91] A. F. Beardon, Iteration of rational functions, Complex analytic dynamical systems, Graduate Texts in Mathematics, 132, Springer-Verlag, New York, 1991.

[Ber93] W. Bergweiler, Iteration of meromorphic functions, Bull. Amer. Math. Soc. 29 (1993), 151-188.

[BH99] W. Bergweiler and A. Hinkkanen, On semiconjugation of entire functions, Math. Proc. Cambridge Philos. Soc. 126 (1999), no. 3, 565-574.

[Bar08] K. Barański, Hausdorff dimension of hairs and ends for entire maps of finite order, Math. Proc. Cambridge Philos. Soc. 145 (2008), no. 3, 719-737.

[CG93] L. Carleson and T. W. Gamelin, Complex dynamics, Universitext: Tracts in Mathematics, Springer-Verlag, New York, 1993.

[DG87] R. L. Devaney and L. R. Goldberg, Uniformization of attracting basins for exponential maps, Duke Math. J. 55 (1987), 253-266.

[DK84] R. L. Devaney and M. Krych, Dynamics of exp(z), Ergod. Th. Dynam. Sys. 4 (1984), $35-52$.

[DT86] R. L. Devaney and F. Tangerman, Dynamics of entire functions near the essential singularity, Ergod. Th. Dynam. Sys. 6 (1986), 489-503.

[Ere89] A. E. Eremenko, On the iteration of entire functions, Dynamical systems and ergodic theory (Warsaw, 1986), 339-345, Banach Center Publ., 23, PWN, Warsaw, 1989.

[EL92] A. E. Eremenko and M. Yu. Lyubich, Dynamical properties of some classes of entire functions, Ann. Inst. Fourier (Grenoble) 42 (1992), no. 4, 989-1020.

[Evd16] V. Evdoridou, Fast escaping points of entire functions: a new regularity condition, Math. Proc. Cambridge Philos. Soc. 160 (2016), no. 1, 95-106.

[Fat26] P. Fatou, Sur l'itération des fonctions transcendantes entières, Acta Math. 47 (1926), 337-370.

[Kar99a] B. Karpińska, Area and Hausdorff dimension of the set of accessible points of the Julia sets of $\lambda e^{z}$ and $\lambda \sin (z)$, Fund. Math. 159 (1999), 269-287.

[Kar99b] B. Karpińska, Hausdorff dimension of the hairs without endpoints for $\lambda \exp z, C$. R. Acad. Sci. Paris Sér. I Math. 328 (1999), 1039-1044.

[Mis81] M. Misiurewicz, On iterates of $e^{z}$, Ergod. Th. Dynam. Sys. 1 (1981), 103-106.

[McM87] C. T. McMullen, Area and Hausdorff dimension of Julia sets of entire functions, Trans. Amer. Math. Soc. 300 (1987), no. 1, 329-342.

[Mil06] J. Milnor, Dynamics in One Complex Variable: Third Edition, Annals of Mathematics Studies, 160, Princeton Univ. Press, Princeton, NJ, 2006.

[Qiu94] W. Qiu, Hausdorff dimension of the $M$-set of $\lambda \exp (z)$, Acta Math. Sinica (N.S.) 10 (1994), 362-368.

[Rem14] L. Rempe, Hyperbolic entire functions with full hyperbolic dimension and approximation by Eremenko-Lyubich functions, Proc. Lond. Math. Soc. 108 (2014), no. 5, 1193-1225.

[RS10] L. Rempe and G. M. Stallard, Hausdorff dimensions of escaping sets of transcendental entire functions, Proc. Amer. Math. Soc. 138 (2010), 1657-1665.

[RS12] P. J. Rippon and G. M. Stallard, Fast escaping points of entire functions, Proc. Lond. Math. Soc. 105 (2012), no. 4, 787-820.

[Sch07] D. Schleicher, The dynamical fine structure of iterated cosine maps and a dimension paradox, Duke Math. J. 136 (2007), 343-356.

[Sch08] H. Schubert, Area of Fatou sets of trigonometric functions, Proc. Amer. Math. Soc. 136 (2008), no. 4, 1251-1259.

[Six11] D. J. Sixsmith, Entire functions for which the escaping set is a spider's web, Math. Proc. Cambridge Philos. Soc. 151 (2011), no. 3, 551-571.

[Six13] D. J. Sixsmith, On fundamental loops and the fast escaping set, J. Lond. Math. Soc. 88 (2013), no. 3, 716-736.

[Six15a] D. J. Sixsmith, Julia and escaping set spiders' webs of positive area, Int. Math. Res. Not. (2015), no. 19, 9751-9774.

[Six15b] D. J. Sixsmith, Functions of genus zero for which the fast escaping set has Hausdorff dimension two, Proc. Amer. Math. Soc. 143 (2015), no. 6, 2597-2612.

[Sta91] G. M. Stallard, The Hausdorff dimension of Julia sets of entire functions, Ergod. Th. Dynam. Sys. 11 (1991), 769-777.

[Tan03] M. Taniguchi, Size of the Julia set of structurally finite transcendental entire function, Math. Proc. Cambridge Philos. Soc. 135 (2003), 181-192.

[ZL12] G. Zhan and L. Liao, Area of non-escaping parameters of the sine family, Houston J. Math. 38 (2012), 493-524. 
Department of Mathematics, Nanjing University, Nanjing, 210093, P. R. China E-mail address: dg1521017@smail.nju.edu.cn

Department of Mathematics, Nanjing University, Nanjing, 210093, P. R. China E-mail address: yangfei@nju.edu.cn 